\title{
Una masa renal poco común en una adolescente - presentación de caso y revisión de la literatura
}

\section{An Unusual Renal Mass in Adolescent - Case Report and Literature Review}

\author{
Jhonatan Sair Santafé Galvis ${ }^{1}$ Dilma Alexandra Cruz Arévalo ${ }^{2}$ Javier Mauricio Salgado Tovar ${ }^{2}$ \\ ${ }^{1}$ Departamento de Urología, Hospital Universitario Mayor, \\ Universidad del Rosario, Bogotá, Colombia \\ 2 Departamento Urología, Universidad Nacional Colombia, Hospital \\ Address for correspondence Jhonatan Sair Santafé Galvis, Residente \\ de Urología, Universidad del Rosario, Bogotá, Colombia \\ (e-mail: john.santafe01@hotmail.com).twitter Id as @JohnS0119.
} Universitario Mayor, Méderi, Bogotá, Colombia

Urol Colomb 2019;28:321-329.

\section{Resumen}

\section{Palabras Clave}

- adenoma metanéfrico

- adolescente

- carcinoma de células renales

- pediatría

- neoplasias renales

- tumor de wilms

\section{Abstract}

Introducción Las neoplasias renales en la población pediátrica y adolescente son raras, entre el $6 \%$ y el $7 \%$ de las masas en pediatría. En adolescentes plantea un desafío clínico importante. Presentan variables manifestaciones sistémicas inespecíficas o cuadros asintomáticos. En la literatura se han identificado el Carcinoma de Células Renales y el Tumor de Wilms como las masas renales más prevalentes en menores de 20 años, el Adenoma Metanéfrico es una entidad muy poco común en esa población.

Presentación de caso Adolescente, con dolor en flanco izquierdo y hematuria macroscópica monosintomática, estudios imagenológicos evidencian lesión de aspecto neoplásico renal izquierda, que al complementarse con estudios de inmunohistoquímica resulta en Adenoma Metanéfrico. Se realiza revisión de literatura publicada en las últimas tres décadas al respecto. La paciente es llevada a nefrectomía radical izquierda, y posteriormente el estudio anatomopatológico sugiere Adenoma Metanéfrico, con marcadores de inmunohistoquímica que corroboran el hallazgo.

Conclusiones Los tumores renales en adolescentes son raros. Se requiere de alta sospecha clínica, un examen físico meticuloso y el apoyo en imágenes diagnósticas. Generalmente, el estudio histopatológico determina el diagnóstico definitivo, no obstante, cuando persiste la confusión, se recurre a la inmunohistoquímica. El Carcinoma de Células Renales es el más prevalente de los tumores renales en adolescentes, pero existen entidades indistinguibles al estudio imagenológico que plantean un desafío clínico; es el objetivo brindar una herramienta de apoyo en el abordaje del Adenoma metanéfrico e instar al desarrollo de conocimiento sobre una entidad patológica en ese grupo etario que ha sido poco documentado que indudablemente redundará en mejores prácticas e impacto científico, social y económico.

Introduction Renal neoplasms in pediatrics and adolescents patients are rare entities, 6-7\% masses in pediatrics. In teenagers, set a noteworthy clinical challenge. Can manifest as variable signs or be asymptomatic. In scientific papers, received

December 31, 2015

accepted

February 8, 2018
DOI https://doi.org/

10.1055/s-0038-1641726.

ISSN 0120-789X.

e ISSN 2027-0119.
Copyright ( 2019, Sociedad Colombiana License terms de Urología. Publicado por Thieme Revinter Publicações Ltda., Rio de Janeiro, Brazil. Todos los derechos reservados.

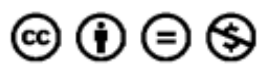




\section{Keywords}

- renal neoplasms

- renal cell carcinoma

- wilms tumor

- pediatrics

- adolescent

- metanephric adenoma
Renal Cell Carcinoma and Wilms' tumor have been identified as the most prevalent tumors in aged lesser than 20 years; Metanephric Adenoma is an unusual pathologic entity into this population.

Case Report A female youngster case, with left flank pain and monosymptomatic macroscopic hematuria, imaging studies show up a neoplastic appearance lesion at left kidney, demonstrated as Metanephric Adenoma afterwards by immunohistochemistry analysis. Literature review is performed by searching in the last three decades. Patient was taken to left radical nephrectomy, later the pathological analysis suggests Metanephric Adenoma, and with immunohistochemistry markers is supported this finding.

Conclusions Renal masses in adolescents are uncommon. It is needed high clinical suspicion, a thorough physical examination and imaging techniques aid. Conventionally, histopathology determine diagnosis, although in some cases when doubt last, it is necessary perform immunohistochemistry. Renal Cell Carcinoma is the most prevalent in youth's renal masses, but there are indistinguishable conditions on imaging that raise a defiant clinical scenario; it is the purpose to provide a clinical guidance tool in the approach of Metanephric adenoma and contribute to the development of knowledge in a scarcely documented clinicopathological entity that doubtlessly will reverberate in best clinical practice and echo at science, social and economics.

\section{Introducción}

Las neoplasias renales en población pediátrica y adolescente son entidades raras, comprenden entre el $6 \%$ y el $7 \%$ de las masas en pediatría. ${ }^{1}$ Raramente ocurren en la segunda década de la vida, menos del $5 \%$ debutan durante la adolescencia. ${ }^{2-5} \mathrm{El}$ abordaje diagnóstico suele estar orientado por establecimiento de grupos etarios. Por ejemplo, se encuentra documentada una mayor prevalencia en jóvenes menores a 15 años del tumor de Wilms (TW), estimada hasta del $87 \%$, aunque con un $80 \%$ correspondiente a menores de 5 años., ${ }^{1,3}$ Por otro lado, en pacientes adultos se considera el Carcinoma de células renales (CCR) como el tumor renal más prevalente. Entre los 11 y 21 años, edad a partir de la cual varias publicaciones consideran como adolescente al individuo, no existe un estudio epidemiológico para determinar la entidad más prevalente, sin embargo, hay publicaciones donde el CCR muestra una preponderancia sobre otras tumoraciones renales en ese grupo etario $^{2}$ además, existe documentación de una tasa de prevalencia directamente proporcional después de los 10 años en esa entidad. ${ }^{6,7}$ De igual manera, hay otras entidades tumorales renales que se pueden presentar durante la adolescencia, dentro de ellas está el Adenoma Metanéfrico (AM), un tumor renal epitelial, raro, considerado benigno y con una primera descripción clinicopatológica amplia publicada en una serie de casos tomados del Instituto de Patología de las Fuerzas Armadas por Davis y col. en 1995, ${ }^{8}$ sin embargo, con similitud anatomopatológica y radiológica con otros tumores de comportamiento conocido maligno como lo son el TW, CCR tipo papilar (pCCR) ${ }^{7-10}$ e incluso se ha descrito la confusión citológica con siembras metastásicas de tiroides y pulmón; ${ }^{11,12}$ lo anterior, aunado a la rareza del cuadro y escasa publicación, crean una necesidad de investigación para determinar el abordaje diagnóstico que permita disminuir la morbilidad ante esa entidad, dado que muchas veces se decide la nefrectomía total o radical en razón de su dificultad diagnóstica. ${ }^{13,14}$

La identificación de esas patologías puede realizarse durante el examen físico de la consulta médica o por un familiar, así como de manera incidental durante procedimientos terapéuticos o diagnósticos. ${ }^{1,14}$ Existe un evidente desafío en la distinción entre masas benignas o tumores malignos con imágenes, determinante en la prioridad del manejo. ${ }^{1,15,16}$ Además, hay muy poca información referente al estudio imagenológico en esa población, ${ }^{3,17}$ lo que conlleva a establecer la relación clínica e imagenológica en el abordaje de esas entidades. El gold standard en el diagnóstico definitivo es el estudio anatomopatológico de la masa renal. ${ }^{1}$

\section{Presentación De Caso}

Adolescente de 17 años de edad, quien ingresa al servicio de Urología en nuestro hospital, con cuadro de 8 días de evolución, caracterizado por dolor en flanco izquierdo de intensidad moderada asociado a hematuria macroscópica, la cual se encontraba en estudios ambulatorios, sin síntomas urinarios irritativos, sin fiebre, sin pérdida de peso. Trae reporte de ecografía renal y vías urinarias donde se encuentra reportado riñón izquierdo aumentado de tamaño por masa sólida que compromete el riñón descrito, además litiasis renal izquierda e hidronefrosis grado II ipsilateral. La paciente presenta antecedente de síndrome anémico desde su niñez y hospitalización 1 año atrás debido a cuadro caracterizado por 
hematuria macroscópica monosintomática, sin seguimiento. Al examen físico, se encuentra estable en sus signos vitales, sin distermia, eutrófica, sensación de masa palpable en flanco izquierdo no dolorosa, sin irritación peritoneal, puñopercusión bilateral negativa. Uroanálisis muestra microhematuria y, hemograma con hemoglobina normal, sin cambios en otras líneas celulares. Imágenes tomográficas simple y contrastada muestran lesión tipo masa hiperdensa heterogénea ( - Fig. 1a y 1b. TAC contrastado de tumor renal. Corte axial), con contornos lobulados, de aspecto neoplásico, localizada en los dos tercios superiores del riñón izquierdo, con calcificaciones gruesas en su interior, diámetros mayores de $144 \times 82 \times 82$ milímetros (-Fig. 1c y 1d. TAC contrastado de tumor renal. Plano coronal). La vena esplénica se encuentra comprimida y disminuida de calibre por efecto de masa, no se aprecia invasión del seno renal izquierdo ni adenopatías retroperitoneales, las demás estructuras abdominales son de aspecto usual (-Fig. 1a-d. TAC contrastado de tumor renal. Corte axial y plano coronal). Se considera como primera posibilidad carcinoma de células renales, se administra manejo sintomático y se programa nefrectomía radical izquierda, la cual se realiza de manera electiva a los 3 meses siguientes de la primera hospitalización. Mediante incisión subcostal izquierda se identifica masa dependiente de tercio medio y superior del riñón izquierdo, con diámetro mayor aproximado de 15 centímetros que distorsiona el parénquima renal, bien demarcada, aspecto amarillento, no infiltrativo, el espécimen es enviado a patología. La paciente egresa en buenas condiciones, con manejo adecuado de dolor y, hasta el momento, ha presentado evolución favorable en su seguimiento postoperatorio (primer año).

\section{Anatomopatología}

Riñón izquierdo con superficie deformada por masa de aproximadamente $14 \times 7 \times 8$ centímetros que involucra polo superior e hilio vascular con dilatación secundaria de cálices. Al corte, se evidencia masa bien delimitada con dimensiones $10.5 \times 9.5 \times 9$ centímetros, de aspecto expansivo, homogéneo, carnoso y color pardo claro. Al estudio bajo microscopía de luz, se evidencia neoplasia delimitada de aspecto embrionario con células pequeñas monomórficas apiñadas en conformación de pseudotúbulos y algunas estructuras ramificadas, nucléolo inconspicuo, no mitosis ni necrosis, se observan calcificaciones tipo psammoma. Se considera espécimen compatible con AM, y se solicita estudio inmunohistoquímico para diferenciar con otras entidades de aspecto embrionario.

\section{Inmunohistoquímica}

En nuestro estudio de patología, se decide realizar el análisis con inmunohistoquímica de un bloque celular, obteniéndose positividad para marcadores AE1/AE3, Vimentina, WT1,
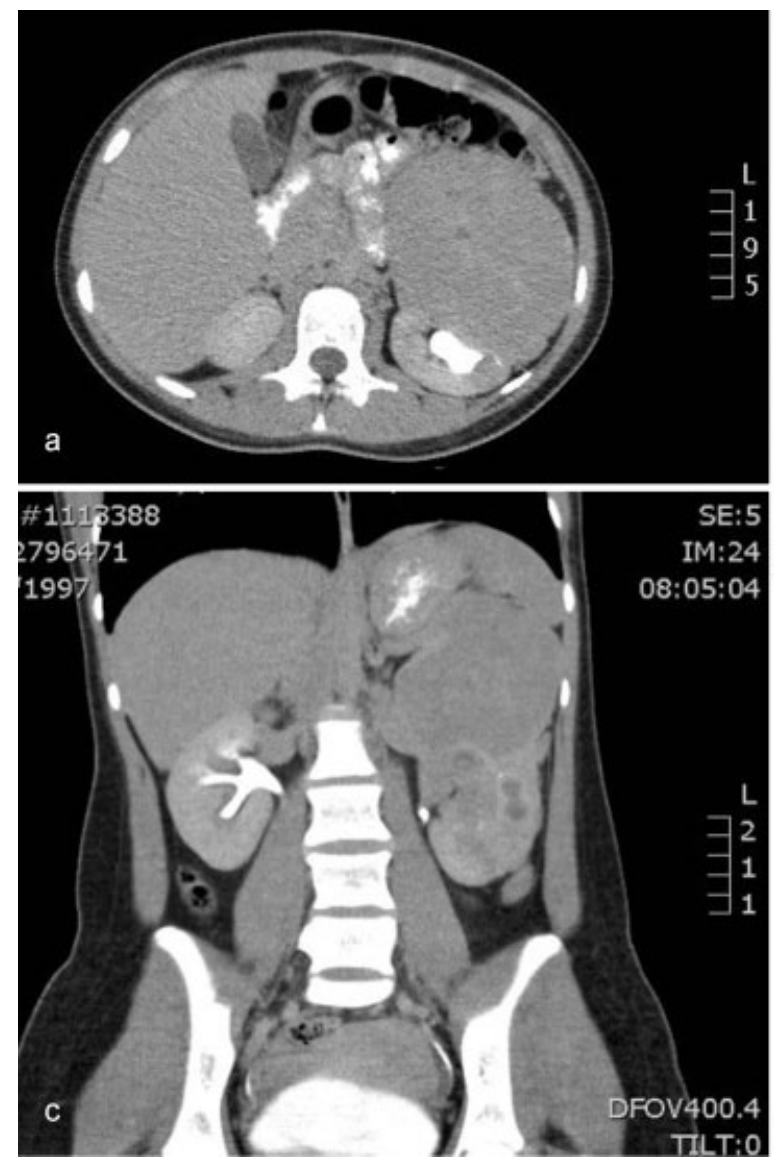
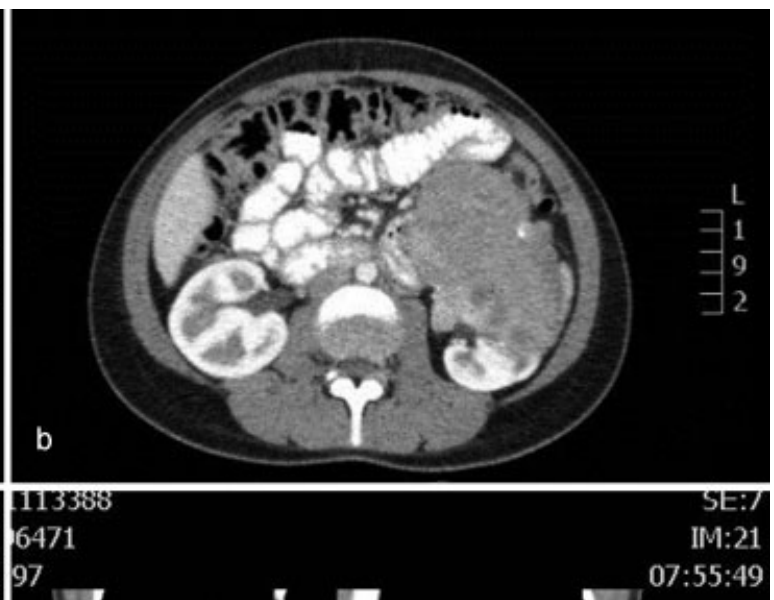

97

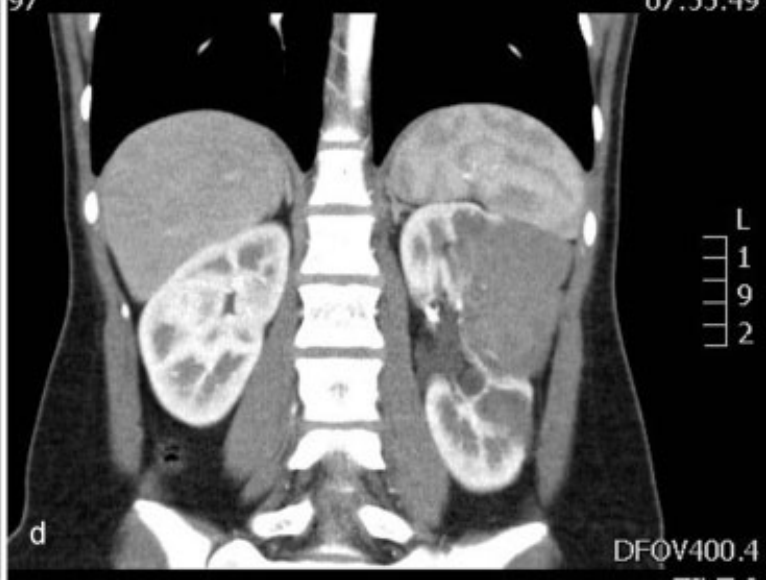

Fig. 1 (a) TAC contrastado de tumor renal. Corte axial. (b) TAC contrastado de tumor renal. Corte axial. (c) TAC contrastado de tumor renal. Plano coronal. (d) TAC contrastado de tumor renal. Plano coronal. 
CD57 y de manera focal para CK7. El índice de proliferación (Ki67) fue bajo con 2\%. Hubo resultados negativos en el estudio con CD99, EMA, sinaptofisina, cromogranina y Fli1. Resultados que son compatibles con Adenoma metanéfrico, lo que descarta el cuadro sospechado inicialmente de CCR.

\section{Discusión}

Las neoplasias renales en la población pediátrica y adolescente son raras. ${ }^{2}$ Existe una clara prevalencia del TW en población infantil menor de 15 años, con pico de presentación antes de los 5 años. En el otro extremo, se encuentra la población adulta, con diagnóstico más frecuente de CCR. ${ }^{18,19}$ No obstante, en población adolescente existe una escasa información epidemiológica que permita definir un predominio neoplásico, por lo que en estudios retrospectivos se han realizado análisis de series de caso, como el caso de Grabowski y colaboradores, quienes mediante el uso del California Cancer Registry realizaron un estudio que arrojó como resultado la existencia de una "transición" durante la adolescencia desde el tumor más prevalente de la infancia (TW) hacia el más diagnosticado en adultos (CCR). El autor concluye, que ante el caso de un tumor renal en paciente entre los 11 y los 20 años de edad, la probabilidad más alta es que se trate de uno de los dos mencionados. ${ }^{2}$ No obstante, se ha visto que hasta el $10-30 \%$ de las masas renales son benignas al momento de la cirugía. ${ }^{20}$ De ahí, la importancia del reporte de nuevos casos que ante la escasa frecuencia y los datos disponibles limitados, ofrecen la oportunidad de conocer mejor la historia natural de la enfermedad, mejorando las intervenciones y calidad de terapéutica. ${ }^{21}$ Un ejemplo, es cómo en el estudio del TW, al evidenciar la ausencia de gran inestabilidad genética como otros cánceres, ha permitido estudiar alteraciones presentes en su citohistopatología y su rol en el desarrollo del cáncer, permitiendo la sobrevida mayor del $85 \%$ que actualmente se tiene para esa neoplasia renal. ${ }^{22}$

El Adenoma Metanéfrico (AM), es una entidad rara, con curso benigno atribuido, ${ }^{23-25}$ que puede originarse de cualquier parte del riñón, predominantemente en la corteza. Representa aproximadamente el $0,2 \%$ de los tumores epiteliales renales en adultos, y no existen datos para adolescentes. ${ }^{26}$ Se ha documentado un amplio rango de edades de presentación que van desde los 15 meses de vida hasta los 83 años, ${ }^{27-29}$ con mayor frecuencia entre la quinta y sexta década de la vida, sin diferencia racial. ${ }^{21}$ Presenta predominio femenino 2:1.21,24 Es una condición poco común en adolescentes que se cree puede estar relacionada con el $\mathrm{TW}^{30}$ por remembranza de los túbulos metanéfricos del riñón fetal, ${ }^{9}$ no obstante de histogénesis aún desconocida, por lo que aún algunos lo plantean como un extremo de mayor diferenciación del TW. ${ }^{30}$ Existen aproximadamente 100 casos descritos en la literatura. ${ }^{31} \mathrm{La}$ primera descripción fue realizada por Brisigotti en 1992, luego complementada por Mostofi y col., en 1988, ${ }^{32,33}$ no obstante existen datos que Bove y col. fue quien realiza la primera descripción en un raro caso bilateral en $1979 .^{28}$ Desde la Clasificación de los Tumores Renales de la Organización Mundial de la Salud en 2004, se clasifica como una entidad distinta con características patológicas, morfogenéticas y de comportamiento biológico único, que además, forma parte de la familia de tumores metanéfricos junto al adenofibroma metanéfrico (AFM) y al tumor estromal metanéfrico (TEM). ${ }^{34-36}$

Su hallazgo es incidental en el $40 \%$ al $60 \%$ de los casos. ${ }^{21} \mathrm{Su}$ presentación puede ser asintomática o con manifestaciones leves. Dentro de los síntomas descritos más prevalentes, se encuentran el dolor en flanco o abdominal (22\%), masa palpable abdominal (10\%-15\%), hematuria macroscópica $(10 \%-27 \%),{ }^{21,37}$ es la neoplasia que posee la relación con el más alto valor de asociación a policitemia $(12 \%),{ }^{21}$ eso al parecer relacionado con la mayor producción de eritropoyetina (EPO) o una sustancia similar a la EPO y algunas interleucinas tales como IL6, IL8 y GM-CSF que favorecen la eritrogénesis, ${ }^{38-40}$ además puede relacionarse a otros síntomas paraneoplásicos tales como hipertensión, disuria, fiebre, astenia, hipercalcemia. ${ }^{21,41}$ Existe un caso con quiluria. ${ }^{42}$

Es un tumor de tamaño variable, unilateral (aunque se han descrito casos de presentación bilateral), ${ }^{8,43,44}$ sólido, homogéneo, con o sin cápsula fibrosa. Con presencia de calcificaciones en aproximadamente el $20 \%$ de los casos. ${ }^{16}$ No posee lateralidad predominante. ${ }^{37}$ En el estudio macroscópico generalmente se aprecia una lesión caféamarillenta, homogénea, con diferenciación del parénquima renal normal bien delimitado, puede contener áreas de hemorragia, necrosis o degeneración quística, igualmente puede presentar calcificaciones multifocales.

Radiológicamente el AM es de compleja distinción al pCCR o a TW. ${ }^{34}$ Con muy pocos rasgos característicos identificados de esa entidad, dado que la mayoría de reportes disponibles están en el área de patología y pocos hallazgos radiológicos se han publicado, ${ }^{37}$ lo que limita la precisión diagnóstica preoperatoria. En el estudio con ecografía pueden observarse lesiones hiperecoicas, ${ }^{28,32,45}$ isoecoicas $^{46}$ o hipoecoicas. $^{33,47}$ En tomografía (TAC) se observan lesiones sólidas, bien circunscritas, unilaterales, homogéneas, sin evidencia de metástasis, con menor realce o igual densidad al parénquima adyacente, posteriormente con la administración del contraste se observa hipodenso en todas las fases, e incluso se ha mencionado la característica de "realce lento progresivo"23,48,49 dado la composición de la masa con menor densidad de microvasculatura (MVD). Del mismo modo, se ha descrito la presentación como lesión hiperdensa atribuido al alto ratio núcleo-citoplasma característico en la histología de ese tumor. ${ }^{45}$ En estudios con doppler se ha observado que esas lesiones suelen ser hipovascularizadas. ${ }^{13}$ En ocasiones, se puede demostrar una imagen de masa hiperdensa, con leve realce con el material de contraste, pero hipodensa respecto al tejido renal adyacente. ${ }^{50} \mathrm{La}$ dificultad diagnóstica con las imágenes resulta de la similitud que pueden compartir con el TW y el pCCR: una imagen con leve hiperatenuación en la tomografía sin contraste, y posterior a su administración, un realce leve, pero que continua siendo menor comparado con el tejido renal adyacente. ${ }^{50}$ Zhu y colaboradores proponen una descripción del AM como una lesión solitaria, pobremente 
circunscrita, isodensa ${ }^{23}$ o hipodensa, con realce menor a la corteza renal y a la médula en todas las fases. ${ }^{28}$ Existen pocos estudios en resonancia magnética disponibles que reportan hipointensidad en Ty T2, incluso post contraste, explicado por la composición del tumor y su hipovascularidad. ${ }^{45}$

Histológicamente, el AM se reporta como células pequeñas monomórficas en configuración tubular o algunas conformaciones glomeruloides, ${ }^{30}$ cuboides o columnares..$^{51}$ Por lo general, no presenta encapsulamiento. Suelen encontrarse calcificaciones o cuerpos de psammoma y una menor tasa mitótica comparada con el CCR tipo papilar ${ }^{9}$ o el TW. Las células pueden distribuirse en diferentes patrones (papilar, tubular, acinar o glomerular), siendo el tubular el más frecuentemente identificado en adolescentes. ${ }^{13,52}$ Presentan núcleo redondo u oval, formación de proyecciones papilares conocidas como cuerpos glomeruloides, escaso citoplasma, estroma escaso o hialino, puede visualizarse degeneración quística y formación de cuerpos de psamomma, con figuras mitóticas raras o nulas. $^{51}$

Por otra parte, el Tumor de Wilms (TW), o nefroblastoma, es considerada la malignidad renal pediátrica más común, con una prevalencia entre $6 \%$ y $7 \%$ en menores a 20 años, ${ }^{53}$ con un pico de presentación entre los 2 y los 5 años de edad. ${ }^{1}$ La mayoría de los casos son esporádicos, pero existe una clara evidencia de asociación con síndromes genéticos que predisponen al desarrollo de TW, algunos de herencia autosómica dominante, entre los que se pueden destacar: sindrome WAGR (TW, aniridia, anomalías genitourinarias y déficit cognitivo), síndrome Denys-Drash, síndrome de sobrecrecimiento (Overgrowth), aniridia esporádica, hemihipertrofia aislada. ${ }^{54}$ Igualmente, se ha descrito asociación con mutaciones tipo del11p13 y mutaciones sin sentido en $W T 11^{1,55}$ Puede manifestarse con síntomas característicos de dolor abdominal, sensación de masa, distensión abdominal, así como hipertensión arterial o simplemente no generar sintomatología. En imágenes es descrito como masa intrarrenal, con ecogenicidad variable debido a la presencia de calcificaciones, necrosis o hemorragias dado su mayor crecimiento comparativo con AM y CCR. Suele describirse como lesión hipodensa o isodensa en el estudio tomográfico simple, con poco o variable realce con el medio de contraste. En el estudio histopatológico, se aprecia patrón embrionario trifásico con componentes blastémicos, epiteliales y estromales. Con difícil diferenciación de otras entidades como el CCR de histología papilar o el adenoma metanéfrico cuando la histología del TW presenta predominio epitelial. ${ }^{1}$

Finalmente, el Carcinoma de células renales (CCR), representa menos del 0,3\% de los tumores en niños, no obstante es el más común en adultos y se ha documentado en la literatura una elevación directamente proporcional de su incidencia a medida que incrementa la edad. ${ }^{1,4,6}$ Su pico de incidencia se encuentra cercano a los 17 años. $^{2}$ Entre el 0,4\% y el $1,3 \%$ de los CCR ocurre en $<15$ años. ${ }^{56-58}$ En adultos, parece preponderar en el sexo masculino. Sin embargo, en niños y adolescentes, esa relación no queda clara. ${ }^{2,59}$ Existen unos factores de riesgo descritos tales como el tabaquismo, la obesidad, y algunos factores ocupacionales. ${ }^{60}$
El CCR es una tumoración epitelial del túbulo renal, y se ha venido asociando con condiciones genéticas como el síndrome de von Hippel-Lindau (VHL), la esclerosis tuberosa (ET), enfermedades hereditarias del tipo carcinoma renal de células claras familiar y carcinoma renal papilar familiar, también se ha hallado como neoplasia en pacientes con enfermedad renal terminal, enfermedad renal quística, hemoglobinopatías, receptores de trasplante renal e incluso en algunos sobrevivientes a cánceres como la leucemia, tumores neuroectodérmicos supratentoriales, síndrome BirtHogg-Dube, TW y neuroblastoma. ${ }^{1,61}$ Se han descrito translocaciones a nivel de Xp11.2, ${ }^{62}$ que compromete a un factor de transcripción denominado TFE3, así como 6p21, donde se afecta otro gen regulador conocido como TFEB. ${ }^{6} \mathrm{La}$ presentación en adolescentes puede debutar asintomática (hasta en el 12\%), y se ha documentado que en adultos jóvenes y adolescentes, el CCR de tipo papilar es el más frecuente. $^{19}$ Se ha descrito una triada (Grawitz) que comprende la presencia de dolor en flanco, masa palpable y hematuria macroscópica indolora, empero mostrando una baja incidencia. ${ }^{63}$ Aronson y colaboradores, en 1996 encontraron que sólo el $9 \%$ presentaban esa tríada en un análisis retrospectivo de 22 pacientes. ${ }^{64}$ La diferenciación con otras neoplasias renales requiere de estudio inmunohistoquímico, y las imágenes por tomografía computarizada (TC) con frecuencia demuestran una masa heterogénea, circunscrita o no, con menor densidad respecto al tejido renal normal adyacente, con frecuencia de calcificaciones hasta el $25 \%$ de los casos y de mayor tamaño que el AM. ${ }^{65}$ Puede presentar invasión vascular, y se le puede encontrar envuelta en una pseudocápsula que en ocasiones se calcifica. ${ }^{1,61}$ En MRI se observa baja señal en Ty T2, dado a que el pCCR posee menor MVD, asimismo, posterior al contraste, se intensifica menos que el tejido renal adyacente. ${ }^{18}$ En la literatura, el rasgo característico del CCR en población adolescente es la mayor incidencia de compromiso nodular linfático regional al momento del diagnóstico. Por lo tanto, algunos datos sugieren que la intervención quirúrgica con resección de ganglios linfáticos regionales debe ser un abordaje de primera línea, asociado o no a la quimioterapia. ${ }^{7}$ Por eso, al momento del diagnóstico, puede encontrarse diseminación extrarrenal del tumor, con enfermedad nodular regional que ha sido reportada en algunos casos de RCC tipo papilar como AM dado su semejanza histológica. ${ }^{9,15}$

Lo anterior, aunado con el incompleto conocimiento dilucidado de la historia natural o factores pronósticos en cánceres renales en adolescentes, el valor limitado de las imágenes preoperatorias para un diagnóstico definitivo, y la necesidad del estudio inmunohistoquímico para determinar el tipo de tumor, hace que la intervención quirúrgica como primera instancia se encuentre sustentado en la actuación frente a ese escenario, ${ }^{66}$ aún ante la posibilidad de un cuadro benigno. ${ }^{2}$ Se ha planteado estudio con biopsia por aspirado de aguja fina guiado por imágenes, sin embargo, no está protocolizado ni se tiene un perfil de seguridad conocido, y existe el riesgo de diseminación o siembra pasiva a nódulos perirrenales. ${ }^{31,67,68}$ Tampoco ha mostrado buen rendimiento diagnóstico. ${ }^{69}$ 
Así pues, en la evaluación preoperatoria durante la práctica clínica de las masas renales en adolescente, el AM es de difícil diferenciación respecto a otros tumores malignos tales como TW y pCCR. El método quirúrgico se convierte en la herramienta a evaluar para el manejo de esos casos, dado que el diagnóstico definitivo será dado por la patología de la lesión, en asociación con el estudio inmunohistoquímico. Esa decisión puede encontrarse particularmente para esa entidad, entre la resección mediante una técnica conservadora (nefrectomía parcial, conservadora de nefronas, enucleación tumoral) o la nefrectomía radical (evaluando en casos particulares la resección o estudio de ganglios perirrenales o retroperitoneales), dependiente de la localización, el tamaño, las características imagenológicas particulares destacadas de la lesión y la capacidad del urólogo. Lo mencionado, debe tener en cuenta el impacto en la salud cardiovascular, en las expectativas de recuperación y el seguimiento esperado del paciente. ${ }^{28}$ También se ha descrito el seguimiento y manejo conservador, pero la mayoría de estudios no cuentan con periodos de tiempo lo suficientemente largos para establecer un lineamiento con seguridad. ${ }^{70}$ Adicionalmente, existen reportes aislados donde se ha documentado la capacidad de malignidad ${ }^{16}$ y metástasis del AM. ${ }^{71,72}$ Esos casos atípicos o con células carcinomatosas, ${ }^{71-73}$ obligan a replantearse el curso benigno del AM, que no puede ser visto como una lesión absolutamente benigna per se; el pronóstico debe basarse en el seguimiento cuidadoso y el tiempo desde la identificación de la lesión hasta su estudio para obtener un diagnóstico histológico, que permite actuar oportunamente dado su desconocida histogénesis. ${ }^{37,44,74}$

En ese orden de ideas, el uso de marcadores inmunohistoquímicos resulta de notable ayuda y se convierten en valiosas herramientas para lograr un diagnóstico acertado. Existe la descripción inmunohistoquímica realizada para diferenciar un AM de un CCR tipo papilar y del TW de predominio epitelial, ${ }^{11,66}$ adaptada y configurada en el presente documento como aporte para el estudio de esa entidad (-Tabla 1. Marcadores en inmunohistoquímica descritos en TW, CCR y AM). Kinney y col., realizó un estudio con 37 AM (posteriormente reclasificados como pCCR), 13 pCCR y 20 TW donde plantea un patrón esperado para el AM así: CK7 negativo, AMACR negativo, WT1 positivo y CD57 positivo, ${ }^{12}$ asimismo plantean el FISH (Inmunohibridización in situ) como adyuvante para evaluar características que permiten descartar patologías como pCCR cuando exista evidencia alteraciones citogenéticas como ganancia o trisomía en cromosomas 7 y 17

Tabla 1 Marcadores en inmunohistoquímica descritos en TW, CCR y AM

\begin{tabular}{|c|c|c|c|}
\hline & Tumor de Wilms (TW) & $\begin{array}{l}\text { Carcinoma de células renales } \\
\text { (CCR) }\end{array}$ & Adenoma metanéfrico (AM) \\
\hline $\begin{array}{l}\text { Epidemiología / } \\
\text { Clínica }\end{array}$ & $\begin{array}{l}\text { - Prevalencia } 6 \% \text { a } 7 \% \text { en } \\
\text { menores de } 20 \text { años. } \\
\text { - Clara asociación con } \\
\text { síndromes genéticos } \\
\text { predisponentes. } † \\
\text { - Dolor o masa abdominal, } \\
\text { hipertensión, distensión. } \ddagger \\
\text { - Maligno. }\end{array}$ & $\begin{array}{l}\text { - Prevalencia exacta en } \\
\text { adolescentes desconocida } \\
\text { (aprox. 0.4-1.3\%). } \\
\text { - Asociación con síndromes. } \\
\text { genéticos predisponentes. } \diamond \\
\text { - Asintomática. } \\
\text { - Triada de Grawitz (9\%). } \\
\text { - Maligno. }\end{array}$ & $\begin{array}{l}\text { - Prevalencia aproximada } \\
\quad<0.2 \% \\
\text { - Relación hombre: mujer 1:2. } \\
\text { - Asintomática. } \\
\text { - Policitemia (12\%), } \\
\text { hipertensión, hematuria, } \\
\text { masa palpable. } \\
\text { - Benigno* }\end{array}$ \\
\hline $\begin{array}{l}\text { Imágenes } \\
\text { (TAC contrastado) }\end{array}$ & $\begin{array}{l}\text { Gran masa intrarrenal, desplaza } \\
\text { estructuras circundantes. Igual } \\
\text { o menor densidad a tejido renal } \\
\text { normal. Calcificaciones, realce } \\
\text { en parches, invasión vascular y } \\
\text { sistema colector. }\end{array}$ & $\begin{array}{l}\text { Imagen grande heterogénea, } \\
\text { bien o mal circunscrita, menos } \\
\text { realce comparado con } \\
\text { parénquima adyacente. } \\
\text { Pseudocápsula con o sin } \\
\text { calcificación, invasión vascular. }\end{array}$ & $\begin{array}{l}\text { Lesión hiper o isodensa, con } \\
\text { poco realce a la administración } \\
\text { del medio de contraste, aun así, } \\
\text { siendo menor comparado con el } \\
\text { tejido renal adyacente. }\end{array}$ \\
\hline Anatomopatología & $\begin{array}{l}\text { Células pequeñas hipercrómicas } \\
\text { con nucléolo prominente, } \\
\text { escaso citoplasma, figuras } \\
\text { mitóticas frecuentes. } \\
\text { Componentes estromal, } \\
\text { blastémico y epitelial, en } \\
\text { diferentes grados. }\end{array}$ & $\begin{array}{l}\text { Células monomorfas pequeñas } \\
\text { con escaso citoplasma } \\
\text { eosinofílico, en distribución de } \\
\text { túbulos, acinar, láminas o } \\
\text { estructura glomerular. }\end{array}$ & $\begin{array}{l}\text { Células epiteliales pequeñas } \\
\text { monomórficas en patrón } \\
\text { tubular, acinar, glomeruloide o } \\
\text { papilar. Usualmente, no } \\
\text { pseudocápsula, presencia de } \\
\text { calcificaciones tipo psammoma } \\
\text { y baja tasa mitótica. }\end{array}$ \\
\hline Inmunohistoquímica & $\begin{array}{l}\text { WT1, CD56, CD57. } \\
\text { Negatividad para AE1, desmina, } \\
\text { CK7. }\end{array}$ & $\begin{array}{l}\text { CK7 (patrón difuso), AMACR, } \\
\text { TFE3, EMA. } \\
\text { Negatividad para WT1. }\end{array}$ & $\begin{array}{l}\text { CD57 (difuso y fuerte), WT1, } \\
\text { proteína S100, AE1/AE3, CK7 } \\
\text { (patrón focal). }\end{array}$ \\
\hline
\end{tabular}

Fuente: elaboración propia.

$\dagger$ WAGR, Denys-Drash, Beckwith-Wiedemann, aniridia esporádica, hemihipertrofia aislada.

$\ddagger$ El curso asintomático también ha sido descrito.

$\diamond$ von Hippel-Lindau, esclerosis tuberosa, carcinomas renales de tipo familiar. También en enfermedad renal crónica, enfermedad renal poliquística, anemia falciforme, receptores de trasplante renal, sobrevivientes de neoplasias tipo leucemia, PNET supratentoriales, síndrome Birt-Hogg-Dube, TW y neuroblastoma.

*Reportes de enfermedad metastásica con pobre pronóstico.

П Diferentes patrones de inmunohistoquímica descritos para algunos marcadores en la misma entidad. 
o monosomia del cromosoma Y, los cuales han sido hallazgos consistentes exclusivos en pCCR, ausente en AM y TW. ${ }^{12,75}$ En TW la búsqueda de otras alteraciones como pérdida de 11 y $16 q$ o ganancia de 1q y 7q, permiten mejorar el rendimiento de esas pruebas complementarias. La mayoría de neoplasias se logran diagnosticar mediante el análisis de la morfología del tumor y tinciones básicas, no obstante existe un fenómeno de solapamiento entre entidades benignas y malignas, y por esa razón, el uso de inmunohistoquímica adicional es necesaria en algunas ocasiones para mejorar el rendimiento diagnóstico. Precisamente, nuevas moléculas se han usado para el estudio en situaciones de particular complejidad como en la diferenciación de esas tres neoplasias renales: AMACR (enzima mitocondrial y peroxisomal alfa metil CoA racemasa) positiva en hasta un 96\% de pCCR (menor prevalencia en otros subtipos), ${ }^{75,76}$ mutaciones en exón 15 del gen BRAF (serinatreonina que regula vía de señalización MAPK) como V600E (descrita en estudios hasta en el 90\% de las muestras de AM), V600D y K601L que generan pérdida del control de crecimiento celular y senescencia, encontrando además una asociación con un patrón acinar histológico y edad mayor de presentación. ${ }^{77,78}$ Se ha estudiado la presencia de mutaciones o aumento en expresión de receptores de estrógenos o progesterona dado la prevalencia mayor en mujeres, sin embargo, con resultados negativos. $^{28}$

\section{Conclusión}

En conclusión, se puede denotar un avance significativo en las últimas tres décadas respecto al estudio y reconocimiento de los tumores renales en adolescentes, particularmente la diferenciación entre entidades que expresan un perfil histopatológico y fenotípico similar, con una probable asociación histogenética aún sin poder dilucidar, de valor en el abordaje de esas entidades por las implicaciones en el tratamiento y el pronóstico de esos pacientes. Se requiere un estudio de buena calidad epidemiológica y con una muestra representativa de la población propia para poder determinar un protocolo certero y de sensibilidad que permita estudiar en pacientes con masas renales la naturaleza benigna o maligna de la lesión, así como se debe reconocer la importancia de las ayudas diagnósticas que permiten aproximarse al componente histológico como bien se veía en la revisión dado el reflejo en la imagen radiológica de la composición tisular. Asimismo, la inmunohistoquímica debe estar concomitante, debido a la evidencia que soporta dichos hallazgos a su vez consistentes con moléculas expresadas según el tipo histológico, que permiten determinar una orientación e incluso culminan comprobando la sospecha diagnóstica inicial. Nuevas moléculas con mejor rendimiento en especificidad $\mathrm{y}$ sensibilidad se requieren, dado que las actuales han demostrado resultados variables (no obstante con buena reproducibilidad) con planteamiento controversial, y como se evidencia en la revisión de la literatura, existe variabilidad en "patrones esperados" de tinción en esos estudios para un mismo tipo de cáncer o masa renal. Cabe resaltar, que la mayor fuente de información bibliográfica científica que evidencia el gran avance en esa área de la urología, recae en las técnicas de inmunohistopatología, aun no siendo un método para diagnóstico concluyente, y así pues, se recalca la necesidad de instaurar un lineamiento para el abordaje imagenológico que permita compilar información útil para el análisis en esos escenarios o crear un estándar (que permita la particularidad en los casos que lo requieran), el seguimiento necesario que necesita el AM dado su comportamiento incierto, que aunque usualmente benigno, no escapa a los individuales reportes de capacidad metastásica e histología mixta maligna. Además, nos permitimos resaltar, la importancia que tiene la consejería genética en esos casos debido a las asociaciones claras con translocaciones o mutaciones monogénicas, en diagnósticos diferenciales del AM y el apoyo en estudios citogenéticos. De igual forma, el llamado a cada urólogo o médico que se enfrente a un posible Adenoma Metanéfrico, es evaluar cuidadosamente el siguiente paso, dado que en ese tipo de patología lo deseable es la menor invasión y menor compromiso de la función renal que puede acarrear demás situaciones mórbidas, en el contexto de una entidad que ha mostrado evidencia de poder ser manejada con la terapia más conservadora posible. No obstante, sin dejar las alertas, porque como se evidencia es aún difícil la diferenciación con 2 entidades de conocimiento científico por su capacidad maligna. El acompañamiento durante el manejo y el seguimiento del paciente adolescente con neoplasia renal, implica una serie de intervenciones y cambios inesperados, por lo que se hace necesario un abordaje multidisciplinario en esos casos. Los tumores renales en adolescentes son una entidad de baja prevalencia, que pueden cursar asintomáticos o dar muy pocas manifestaciones, el AM es de curso benigno, raro y con sintomatología que puede llegar a ser orientadora, sin embargo, existe poca información en este grupo etario; la extrapolación de series de casos en niños y adultos requiere de mayor cautela y análisis según las herramientas disponibles en cada institución, dado que puede presentarse un matiz de grises en las entidades de ambos extremos de la vida. Se requiere de alta sospecha clínica, un examen físico meticuloso y el apoyo en imágenes, realizar una correlación clínica e imagenológica. El estudio histopatológico determina el diagnóstico definitivo, sin embargo, se debe tener en cuenta la existencia de entidades indistinguibles dentro del espectro de las masas renales en adolescentes, por lo que, la optimización del abordaje diagnóstico con el apoyo en la inmunohistoquímica y la citogenética, es determinante en la terapéutica y seguramente en el pronóstico del paciente. Se hace necesario la evaluación del manejo conservador en esos pacientes no obstante, debe hacerse, cuando se evalúen nuevas técnicas de detección temprana diagnóstica que disminuyan el riesgo de una intervención subterapeútica.

\section{Responsabilidades Éticas}

Protección de personas y animales. Los autores declaran que para esta investigación no se han realizado experimentos en seres humanos ni en animales.

Confidencialidad de los datos. Los autores declaran que han seguido los protocolos de su centro de trabajo sobre la publicación de datos de pacientes. 


\section{Conflicto de Intereses}

Los autores declaran no tener ningún conflicto de intereses.

\section{Referencias}

1 Malkan AD, Loh A, Bahrami A, et al. An approach to renal masses in pediatrics. Pediatrics 2015;135(01):142-158

2 Grabowski J, Silberstein J, Saltzstein SL, Saenz N. Renal tumors in the second decade of life: results from the California Cancer Registry. J Pediatr Surg 2009;44(06):1148-1151

3 Kumar S, Sharma P, Pratap J, Tiwari P, Bera MK, Kundu AK. Renal cell carcinoma in children and adolescence: our experience. Afr J Paediatr Surg 2014;11(02):101-104

4 Ahmed HU, Arya M, Levitt G, Duffy PG, Mushtaq I, Sebire NJ. Part I: Primary malignant non-Wilms' renal tumours in children. Lancet Oncol 2007;8(08):730-737

5 Broecker B. Non-Wilms' renal tumors in children. Urol Clin North Am 2000;27(03):463-469, ix ix

6 Young EE, Brown CT, Merguerian PA, Akhavan A. Pediatric and adolescent renal cell carcinoma. Urol Oncol 2016;34(01):42-49

7 Akhavan A, Richards M, Shnorhavorian M, Goldin A, Gow K, Merguerian PA. Renal cell carcinoma in children, adolescents and young adults: a National Cancer Database study. J Urol 2015;193(04):1336-1341

8 Davis CJ Jr, Barton JH, Sesterhenn IA, Mostofi FK. Metanephric adenoma. Clinicopathological study of fifty patients. Am J Surg Pathol 1995;19(10):1101-1114

9 Muir TE, Cheville JC, Lager DJ. Metanephric adenoma, nephrogenic rests, and Wilms' tumor: a histologic and immunophenotypic comparison. Am J Surg Pathol 2001;25(10):1290-1296

10 Pins MR, Jones EC, Martul EV, Kamat BR, Umlas J, Renshaw AA. Metanephric adenoma-like tumors of the kidney: report of 3 malignancies with emphasis on discriminating features. Arch Pathol Lab Med 1999;123(05):415-420

11 Mantoan Padilha M, Billis A, Allende D, Zhou M, Magi-Galluzzi C. Metanephric adenoma and solid variant of papillary renal cell carcinoma: common and distinctive features. Histopathology 2013;62(06):941-953

12 Kinney SN, Eble JN, Hes O, et al. Metanephric adenoma: the utility of immunohistochemical and cytogenetic analyses in differential diagnosis, including solid variant papillary renal cell carcinoma and epithelial-predominant nephroblastoma. Mod Pathol 2015; 28(09):1236-1248

13 Kuroda N, Tol M, Hiroi M, Enzan H. Review of metanephric adenoma of the kidney with focus on clinical and pathobiological aspects. Histol Histopathol 2003;18(01):253-257

14 Brisigotti M, Cozzutto C, Fabbretti G, Sergi C, Callea F. Metanephric adenoma. Histol Histopathol 1992;7(04):689-692

15 Valera ET, Brassesco MS, de Freitas Cortez MA, et al. Pitfalls in the differential diagnosis of renal tumor in an adolescent. Pediatr Blood Cancer 2010;54(02):319-321

16 Obulareddy SJ, Xin J, Truskinovsky AM, Anderson JK, Franklin MJ, Dudek AZ. Metanephric adenoma of the kidney: an unusual diagnostic challenge. Rare Tumors 2010;2(02):e38

17 Masuda A, Kamai T, Mizuno T, et al. Renal metanephric adenoma mimicking papillary renal cell carcinoma on computed tomography: a case report. Urol Int 2013;90(03):369-372

18 Vikram R, Ng CS, Tamboli P, et al. Papillary renal cell carcinoma: radiologic-pathologic correlation and spectrum of disease. Radiographics 2009;29(03):741-754, discussion 755-757

19 Cao Y, Paner GP, Perry KT, Flanigan RC, Campbell SC, Picken MM. Renal neoplasms in younger adults: analysis of 112 tumors from a single institution according to the new 2004 World Health Organization classification and 2002 American Joint Committee on Cancer Staging System. Arch Pathol Lab Med 2005;129(04): 487-491
20 Le O, Roy A, Silverman PM, Kundra V. Common and uncommon adult unilateral renal masses other than renal cell carcinoma. Cancer Imaging 2012;12:194-204

21 Fajardo GB, Sierra JM, Sánchez EH, López LG, González JB, Moyano ÁS, et al Adenoma metanéfrico y carcinoma de células claras metacrónicos ipsilateral. Madrid, España: Arch. Esp. Urol; 2003: 479-484

22 Coppes MJ, Pritchard-Jones K. Principles of Wilms' tumor biology. Urol Clin North Am 2000;27(03):423-433, viii viii

23 Zhu Q Zhu W, Wu J, Chen W, Wang S. The clinical and CT imaging features of metanephric adenoma. Acta Radiol 2014;55(02):231-238

24 Spaner SJ, Yu Y, Cook AJ, Boag G. Pediatric metanephric adenoma: case report and review of the literature. Int Urol Nephrol 2014;46 (04):677-680

25 Vujanić GM. Renal tumours of childhood: an overview. Diagn Histopathol 2009;15(11):501-509

26 Raman SP, Hruban RH, Fishman EK. Beyond renal cell carcinoma: rare and unusual renal masses. Abdom Imaging 2012;37(05): 873-884

27 Yin M, Cai J, Thorner PS. Congenital renal tumor: metanephric adenoma, nephrogenic rest, or malignancy? Pediatr Dev Pathol 2015;18(03):245-250

28 Bastide C, Rambeaud JJ, Bach AM, Russo P. Metanephric adenoma of the kidney: clinical and radiological study of nine cases. BJU Int 2009;103(11):1544-1548

29 Shek TW, Luk IS, Peh WC, Chan KL, Chan GC. Metanephric adenofibroma: report of a case and review of the literature. Am J Surg Pathol 1999;23(06):727-733

30 Argani P. Metanephric neoplasms: the hyperdifferentiated, benign end of the Wilms tumor spectrum? Clin Lab Med 2005; 25(02):379-392

31 Fan H, Shao QQ, Li HZ, Xiao Y, Zhang YS. The Clinical Characteristics of Metanephric Adenoma: A Case Report and Literature Review. Medicine (Baltimore) 2016;95(21):e3486

32 Bastos Netto JM, Esteves TC, Mattos RdeC, Tibiriçá SH, Costa SM, Vieira LJ. Metanephric adenoma: a rare differential diagnosis of renal tumor in children. J Pediatr Urol 2007;3 (04):340-341

33 Schmelz HU, Stoschek M, Schwerer M, et al. Metanephric adenoma of the kidney: case report and review of the literature. Int Urol Nephrol 2005;37(02):213-217

34 Lara C, Porras V, Jurado P, Arredondo F. Adenoma Metanéfrico. Presentación De Un Caso. Madrid, España: Arch. Esp. Urol; 2007. p. 1.122-1.4

35 Shek TW, Luk IS, Peh WC, Chan KL, Chan GC. Metanephric adenofibroma: report of a case and review of the literature. Am J Surg Pathol 1999;23(06):727-733

36 Argani P, Beckwith JB. Metanephric stromal tumor: report of 31 cases of a distinctive pediatric renal neoplasm. Am J Surg Pathol 2000;24(07):917-926

37 Li G, Tang Y, Zhang R, Song H, Zhang S, Niu Y. Adult metanephric adenoma presumed to be all benign? A clinical perspective. BMC Cancer 2015;15:310

38 Caldwell K, Wilson J, Reyes C. Metanephric adenoma: Management in a 7-year-old child. J Pediatr Surg Case Rep 2015;3(12):570-572

39 Konety BR, Hord JD, Weiner ES, Schneck FX. Embryonal adenoma of the kidney associated with polycythemia and von Willebrand disease. J Urol 1998;160(6 Pt 1):2171-2174

40 Yoshioka K, Miyakawa A, Ohno Y, et al. Production of erythropoietin and multiple cytokines by metanephric adenoma results in erythrocytosis. Pathol Int 2007;57(08):529-536

41 Zhang LJ, Yang GF, Shen W, Lu GM. CT and ultrasound findings of metanephric adenoma: a report of two cases and literature review. Br J Radiol 2011;84(999):e51-e54

42 McNeil JC, Corbett ST, Kuruvilla S, Jones EA. Metanephric adenoma in a five-year-old boy presenting with chyluria: case report and review of literature. Urology 2008;72(03):545-547 
43 Rosero DS, Agua CD, Marquina I, Jalon AGD, Felipo F. Bifocal Metanephric Adenoma in a Patient with Non-hodgkin Lymphoma Carlisle, United Kingdom. Int J Clinical Urology 2017:7-10

44 Pasricha S, Gandhi JS, Gupta G, Mehta A, Beg S. Bilateral, multicenteric metanephric adenoma associated with Wilms tumor in a child: a rare presentation with important diagnostic and therapeutic implications. Int J Urol 2012;19(12):1114-1117

45 Fielding JR, Visweswaran A, Silverman SG, Granter SR, Renshaw AA.CT and ultrasound features of metanephric adenoma in adults with pathologic correlation.J Comput Assist Tomogr 1999;23(03):441-444

46 Hwang SS, Choi YJ. Metanephric adenoma of the kidney: case report. Abdom Imaging 2004;29(03):309-311

47 Navarro O, Conolly B, Taylor G, Bägli DJ. Metanephric adenoma of the kidney: a case report. Pediatr Radiol 1999;29(02):100-103

48 Jinzaki M, Tanimoto A, Mukai M, et al. Double-phase helical CT of small renal parenchymal neoplasms: correlation with pathologic findings and tumor angiogenesis. J Comput Assist Tomogr 2000; 24(06):835-842

49 Hu YC, Wu L, Yan LF, Zhang W, Cui GB. The imaging features of metanephric adenoma: a case report and review of literature. Onco Targets Ther 2015;8:445-449

50 Silverman SG, Mortele KJ, Tuncali K, Jinzaki M, Cibas ES. Hyperattenuating renal masses: etiologies, pathogenesis, and imaging evaluation. Radiographics 2007;27(04):1131-1143

51 Chami R, Yin M, Marrano P, Teerapakpinyo C, Shuangshoti S, Thorner PS. BRAF mutations in pediatric metanephric tumors. Hum Pathol 2015;46(08):1153-1161

52 Sun Z, Kan S, Zhang L, et al. Immunohistochemical phenotype and molecular pathological characteristics of metanephric adenoma. Int J Clin Exp Pathol 2015;8(06):6031-6036

53 Sebire NJ, Vujanic GM. Paediatric renal tumours: recent developments, new entities and pathological features. Histopathology 2009;54(05): 516-528

54 Shamberger RC. Pediatric renal tumors. Semin Surg Oncol 1999; 16(02):105-120

55 Hicks J, Mierau GW. The spectrum of pediatric tumors in infancy, childhood, and adolescence: a comprehensive review with emphasis on special techniques in diagnosis. Ultrastruct Pathol 2005;29(3-4):175-202

56 Taccoen X, Valeri A, Descotes JL, et al; Oncology Committee of the Association Française d'Urologie. Renal cell carcinoma in adults 40 years old or less: young age is an independent prognostic factor for cancer-specific survival. Eur Urol 2007;51(04):980-987

57 Otgün I, Arda IS, Haberal N, Güney H, Hiçsönmez A. Renal cell carcinoma: case report and literature review. J Pediatr Surg 2005; 40(05):e13-e16

58 Spreafico F, Collini P, Terenziani M, Marchianò A, Piva L. Renal cell carcinoma in children and adolescents. Expert Rev Anticancer Ther 2010;10(12):1967-1978

59 Silberstein J, Grabowski J, Saltzstein SL, Kane CJ. Renal cell carcinoma in the pediatric population: Results from the California Cancer Registry. Pediatr Blood Cancer 2009;52(02):237-241

60 Ljungberg B, Campbell SC, Choi HY, et al. The epidemiology of renal cell carcinoma. Eur Urol 2011;60(04):615-621

61 NG C. Jr DW. Renal malignancies in adolescents and young adults. American Urological Association Update Series. 2013;32(26): 257-267
62 Taşkınlar H, Avlan D, Çıtak Ç, Polat A, Naycı A. A rare cause of childhood renal cysts: Xp11.2 translocation renal cell carcinoma. Can Urol Assoc J 2015;9(1-2):E36-E38

63 Estrada CR, Suthar AM, Eaton SH, Cilento BG Jr. Renal cell carcinoma: Children's Hospital Boston experience. Urology 2005;66(06):1296-1300

64 Aronson DC, Medary I, Finlay JL, Herr HW, Exelby PR, La Quaglia MP. Renal cell carcinoma in childhood and adolescence: a retrospective survey for prognostic factors in 22 cases. J Pediatr Surg 1996;31(01):183-186

65 Downey RT, Dillman JR, Ladino-Torres MF, McHugh JB, Ehrlich PF, Strouse PJ. CT and MRI appearances and radiologic staging of pediatric renal cell carcinoma. Pediatr Radiol 2012;42(04): 410-417, quiz 513-514

66 Portugal R, Barroca H. Clear cell sarcoma, cellular mesoblastic nephroma and metanephric adenoma: cytological features and differential diagnosis with Wilms tumour. Cytopathology 2008; 19(02):80-85

67 Paner GP, Turk TM, Clark JI, Lindgren V, Picken MM. Passive seeding in metanephric adenoma: a review of pseudometastatic lesions in perinephric lymph nodes. Arch Pathol Lab Med 2005;129(10): 1317-1321

68 Shet T, Viswanathan S. The cytological diagnosis of paediatric renal tumours. J Clin Pathol 2009;62(11):961-969

69 Chaudhary H, Raghvendran M, Dubey D, et al. Correlation of radiological and clinical features of metanephric neoplasms in adults. Indian J Cancer 2004;41(01):37-40

70 Le Nué R, Marcellin L, Ripepi M, Henry C, Kretz JM, Geiss S. Conservative treatment of metanephric adenoma. A case report and review of the literature. J Pediatr Urol 2011;7(04):399-403

71 Drut R, Drut RM, Ortolani C. Metastatic metanephric adenoma with foci of papillary carcinoma in a child: a combined histologic, immunohistochemical, and FISH study. Int J Surg Pathol 2001;9 (03):241-247

72 Renshaw AA, Freyer DR, Hammers YA. Metastatic metanephric adenoma in a child. Am J Surg Pathol 2000;24(04):570-574

73 Picken MM, Curry JL, Lindgren V, Clark JI, Eble JN. Metanephric adenosarcoma in a young adult: morphologic, immunophenotypic, ultrastructural, and fluorescence in situ hybridization analyses: a case report and review of the literature. Am J Surg Pathol 2001;25 (11):1451-1457

74 Jain M, Rastogi A, Gupta RK. Atypical metanephric adenoma - a case report and review of literature. Int Urol Nephrol 2007;39 (01):123-127

75 Saremian J, Kubik MJ, Masood S. Cytologic features of metanephric adenoma of the kidney: case report and review of the literature. Lab Med 2015;46(02):153-158, quiz e30

76 Olgac S, Hutchinson B, Tickoo SK, Reuter VE. Alpha-methylacylCoA racemase as a marker in the differential diagnosis of metanephric adenoma. Mod Pathol 2006;19(02):218-224

77 Caliò A, Eble JN, Hes O, et al. Distinct clinicopathological features in metanephric adenoma harboring BRAF mutation. Oncotarget 2016;8(33):54096-54105

78 Udager AM, Pan J, Magers MJ, et al. Molecular and immunohistochemical characterization reveals novel BRAF mutations in metanephric adenoma. Am J Surg Pathol 2015;39 (04):549-557 